\title{
Tunable spin-charge conversion through topological phase transitions in zigzag nanoribbons
}

\author{
Hang Li and Aurélien Manchon* \\ King Abdullah University of Science and Technology (KAUST), Physical Science and Engineering Division (PSE), \\ Thuwal 23955-6900, Saudi Arabia
}

(Received 29 April 2016; published 29 June 2016)

\begin{abstract}
We study spin-orbit torques and charge pumping in magnetic quasi-one-dimensional zigzag nanoribbons with a hexagonal lattice, in the presence of large intrinsic spin-orbit coupling. Such a system experiences a topological phase transition from a trivial band insulator to a quantum spin Hall insulator by tuning of either the magnetization direction or the intrinsic spin-orbit coupling. We find that the spin-charge conversion efficiency (i.e., spin-orbit torque and charge pumping) is dramatically enhanced at the topological transition, displaying a substantial angular anisotropy.
\end{abstract}

DOI: 10.1103/PhysRevB.93.235317

\section{INTRODUCTION}

Topological insulators, a new phase of matter, have attracted intense research interest due to their nontrivial physical properties and potential applications in spintronics [1]. Similarly to conventional or band insulators they possess a band gap in the bulk. Yet, differently from conventional insulators they support time-reversal-symmetry-protected spin-polarized surface or edge states in the bulk band gap. These materials may experience a topological phase transition from topological to band insulator by structural design and manipulations such as doping with impurities [2,3], applying a strain or pressure $[4,5]$, and inducing lattice distortion or enhancing spin-orbit coupling via nonmagnetic substrates [6]. Interestingly, even without structural manipulation, a topological phase transition can also be driven by coupling topological insulators with magnetic substrates. For example, a transition from a band insulator to a quantum anomalous Hall insulator can be achieved by inducing a magnetic exchange in silicene via a proximate magnetic layer [7,8]. Among these studies, the influence of topological phase transitions on Hall conductivities and spin textures in momentum space has been confirmed [3,9]. From a topological standpoint, a charge or spin current in a topological insulator is also a topological current. Hence, unlike semiconductors and metals, charge conductivities and spin-polarized edge states in topological insulators can be controlled not only by an electric field but also by topological phase transitions.

Besides topological phase transitions, charges flowing at the surface or edge of topological insulators are accompanied by a nonequilibrium spin polarization due to the large spinmomentum locking of surface states [10]. Such a magnetoelectric effect can be used to excite and switch the magnetization of a ferromagnet deposited on the surface, as studied theoretically $[11,12]$ and demonstrated experimentally [13-15]. This spinorbit torque displays a higher electrical efficiency compared with the spin torque in bilayers involving heavy metals $[16,17]$. Alternatively, the spin-to-charge conversion present at the surface of topological insulators can be probed through charge pumping, i.e., the Onsager reciprocal of spin-orbit torques $[24,25]$. In fact, while a charge current creates a torque on the

\footnotetext{
*aurelien.manchon@kaust.edu.sa
}

magnetization, a precessing magnetization induces a charge current along the interface. This effect was originally observed in magnetic bilayers involving heavy metals and attributed to the inverse spin Hall effect present in the bulk of heavy metals [18]. This observation has recently been extended to two-dimensional systems such as hexagonal lattices [19] and semimetal surfaces [20] and, more recently, to the surface of topological insulators [21-23]. In these systems, the spincharge conversion is attributed to the spin-momentum locking induced by interfacial (Rashba or Dirac) spin-orbit coupling. While magnetoelectric effects have been studied in topological insulators in the metallic regime [11,12], the influence of topological phase transitions on these mechanisms has been essentially overlooked. In particular, besides the emergence of a quantized magnetoelectric effect [10], it is not clear how topologically nontrivial edge states contribute to spin-orbit torque and charge pumping.

In this paper, we theoretically investigate both charge pumping and spin-orbit torque in quasi-one-dimensional zigzag nanoribbons with a hexagonal lattice in the presence of intrinsic spin-orbit coupling and magnetic exchange. Depending on the spin-orbit coupling strength, this system displays topological phase transitions between trivial (metallic) and nontrivial (quantum spin-anomalous Hall) phases [7,33]. We demonstrate that spin-charge conversion efficiency is dramatically enhanced at the topological transition, resulting in large damping-like spin-orbit torque and dc charge pumping.

\section{THEORETICAL PROCEDURE}

\section{A. Spin-orbit torque and charge pumping}

Let us first formulate the reciprocity relationship between spin-orbit torques and charge pumping (see also Refs. [24] and [25]). We start with the definitions of magnetization dynamics and charge current,

$$
\begin{gathered}
\partial_{t} \mathbf{m}=\gamma \mathbf{m} \times \partial_{\mathbf{m}} F+\hat{\chi} \cdot \mathbf{E}, \\
\mathbf{J}_{c}=\hat{\sigma} \cdot \mathbf{E}+\hat{\xi} \cdot \partial_{\mathbf{m}} F,
\end{gathered}
$$

where $-\partial_{\mathbf{m}} F=-\partial_{\mathbf{m}} \Omega / M_{s}$ is the effective field that drives the dynamics of the magnetization in the absence of charge flow. $\gamma$ is the absolute value of the gyromagnetic ratio, $\Omega$ is the magnetic energy density, $\mathbf{m}$ is the unit vector 
of the magnetization direction, and $M_{s}$ is the saturation magnetization. Equation (1) is defined in the absence of magnetic damping, for simplicity. $\mathbf{E}$ is the electric field that drives the charge current through the conductivity tensor $\hat{\sigma}$ in the absence of magnetization dynamics. $\hat{\chi}$ and $\hat{\xi}$ are the tensors accounting for current-driven torques and charge pumping, respectively. We can rewrite these two equations in a more compact form,

$$
\left(\begin{array}{c}
\partial_{t} n_{i} \\
\partial_{t} m_{i}
\end{array}\right)=\left(\begin{array}{cc}
L_{n_{i}, f_{e}^{j}} & L_{n_{i}, f_{m}^{j}} \\
L_{m_{i}, f_{e}^{j}} & L_{m_{i}, f_{m}^{j}}
\end{array}\right)\left(\begin{array}{c}
f_{e}^{j} \\
f_{m}^{j}
\end{array}\right)
$$

where we define the $i$ th component of the particle current $\partial_{t} n_{i}=S \mathrm{~J}_{c, i} / e$ and the $j$ th component of the electric and magnetic forces $f_{e}^{j}=d e E_{j}$ and $f_{m}^{j}=\mu_{B} \partial_{m_{j}} F$, respectively. The Onsager coefficients are then

$$
\left(\begin{array}{cc}
L_{n_{i}, f_{e}^{j}} & L_{n_{i}, f_{m}^{j}} \\
L_{m_{i}, f_{e}^{j}} & L_{m_{i}, f_{m}^{j}}
\end{array}\right)=\left(\begin{array}{cc}
W \sigma_{i j} / e^{2} & \xi^{i j} / \mu_{B} \\
\chi^{i j} / d & -\left(\gamma / \mu_{B}\right)\left(\mathbf{e}_{i} \times \mathbf{e}_{j}\right) \cdot \mathbf{m}
\end{array}\right) .
$$

Here, we consider a magnetic volume of width $W$, thickness $d$, and section normal to the current flow $S=W d$. Applying the Onsager reciprocity principle [24,26],

$$
L_{n_{i}, f_{m}^{j}}(\mathbf{m})=-L_{m_{j}, f_{e}^{i}}(-\mathbf{m}),
$$

and we obtain $\xi^{i j}(\mathbf{m}) / \mu_{B}=-\chi^{j i}(-\mathbf{m}) / d$. In twodimensional magnets with interfacial inversion asymmetry, the spin-orbit torque $\mathbf{T}=\hat{\chi} \cdot \mathbf{E}$ can be parsed into two components (see, e.g., Refs. [12] and [46]),

$$
\mathbf{T}=\tau_{\mathrm{DL}} \mathbf{m} \times((\mathbf{z} \times \mathbf{E}) \times \mathbf{m})+\tau_{\mathrm{FL}} \mathbf{m} \times(\mathbf{z} \times \mathbf{E}),
$$

referred to as the damping-like ( $\left.\tau_{\mathrm{DL}}\right)$ and field-like $\left(\tau_{\mathrm{FL}}\right)$ torque. Hence, by definition,

$$
\begin{aligned}
\chi^{i j}(\mathbf{m})= & \tau_{\mathrm{DL}}\left[\mathbf{m} \times\left(\left(\mathbf{z} \times \mathbf{e}_{j}\right) \times \mathbf{m}\right)\right] \cdot \mathbf{e}_{i} \\
& +\tau_{\mathrm{FL}}\left[\mathbf{m} \times\left(\mathbf{z} \times \mathbf{e}_{j}\right)\right] \cdot \mathbf{e}_{i} .
\end{aligned}
$$

Then, applying Onsager reciprocity, we obtain the charge pumping coefficient

$$
\begin{aligned}
\xi^{i j}(\mathbf{m})= & -\left(\mu_{B} / d\right) \tau_{\mathrm{DL}}(-\mathbf{m})\left[\mathbf{m} \times\left(\left(\mathbf{z} \times \mathbf{e}_{i}\right) \times \mathbf{m}\right)\right] \cdot \mathbf{e}_{j} \\
& +\left(\mu_{B} / d\right) \tau_{\mathrm{FL}}(-\mathbf{m})\left[\mathbf{m} \times\left(\mathbf{z} \times \mathbf{e}_{i}\right)\right] \cdot \mathbf{e}_{j} .
\end{aligned}
$$

And, finally, the charge current induced by the magnetization dynamics reads

$$
\begin{aligned}
\mathbf{J}_{c}= & -\frac{\mu_{B}}{d \gamma} \tau_{\mathrm{DL}}(-\mathbf{m}) \mathbf{z} \times\left(\mathbf{m} \times \partial_{t} \mathbf{m}\right) \\
& +\frac{\mu_{B}}{d \gamma} \tau_{\mathrm{FL}}(-\mathbf{m}) \mathbf{z} \times \partial_{t} \mathbf{m} .
\end{aligned}
$$

This equation establishes the correspondence between the current-driven spin-orbit torque and the charge current pumped by a time-varying magnetization. In the following, we compute the current-driven spin density $\delta \mathbf{S}$ from the Kubo formula [Eq. (11)]. The torque is simply $\mathbf{T}=\left(2 J_{\text {ex }} / \hbar\right) \mathbf{m} \times \delta \mathbf{S}$, so that the conclusions drawn for spin-orbit torques equally apply to charge pumping.

\section{B. Model and method}

Let us now consider a single-layered zigzag nanoribbon with a hexagonal lattice (e.g., silicene, germanene, stanene) deposited on top of a ferromagnetic layer. The ferromagnetic layer may be chosen as $\mathrm{EuO}$ [27] or YIG [19,28] and induces a weak exchange coupling on the spin-polarized carriers as well as Rashba spin-orbit coupling.

In a tight-binding representation, the Hamiltonian for silicene-like material can be described by [29]

$$
\begin{aligned}
\hat{H}_{0}= & \sum_{\langle i, j\rangle} t \hat{c}_{i}^{+} \hat{c}_{j}+i \frac{t_{\mathrm{so}}}{3 \sqrt{3}} \sum_{\langle\langle i, j\rangle\rangle} \hat{c}_{i}^{+} v_{i j} \hat{\mathrm{s}}_{z} \hat{c}_{j} \\
& +i \frac{2 t_{R}}{3} \sum_{\langle i, j\rangle} \hat{c}_{i}^{+} \hat{z} \cdot\left(\hat{\mathbf{s}} \times \mathbf{d}_{i j}\right) \hat{c}_{j}+J_{\mathrm{ex}} \sum_{i} \hat{c}_{i}^{+} \hat{\mathbf{s}} \cdot \mathbf{m} \hat{c}_{i},
\end{aligned}
$$

where $\hat{c}_{i}^{+}=\left(\hat{c}_{i, \uparrow}, \hat{c}_{i, \downarrow}\right)^{+}$, and $\hat{c}_{i, \alpha}^{+}$creates an electron with spin $\alpha$ at site $i .\langle i, j\rangle(\langle\langle i, j\rangle\rangle)$ runs over all the possible nearest-neighbor (next-nearest-neighbor) hopping sites, and $\mathbf{d}_{i j}$ is the position vector between site $i$ and site $j . t_{R}\left(t_{\mathrm{so}}\right)$ is the Rashba (intrinsic) spin-orbit coupling and $J_{\text {ex }}$ is the exchange energy. $\hat{\mathbf{s}}$ is the vector of Pauli spin matrices for spin-1/2 and $v_{i j}= \pm 1$ when the trajectory of electron hopping from site $j$ to site $i$ is anticlockwise (clockwise). The first term denotes the nearest-neighbor hopping, the second term denotes the intrinsic spin-orbit coupling, and the third one represents the extrinsic Rashba spin-orbit coupling. The fourth term is the exchange interaction between the spin of the carrier $\hat{\mathbf{s}}$ and the local moment of the ferromagnet $\mathbf{m}$.

We assume that the nanoribbon is uniform and periodic along the transport direction. A super unit cell is chosen as shown by the red rectangle in Fig. 1(a). To compute
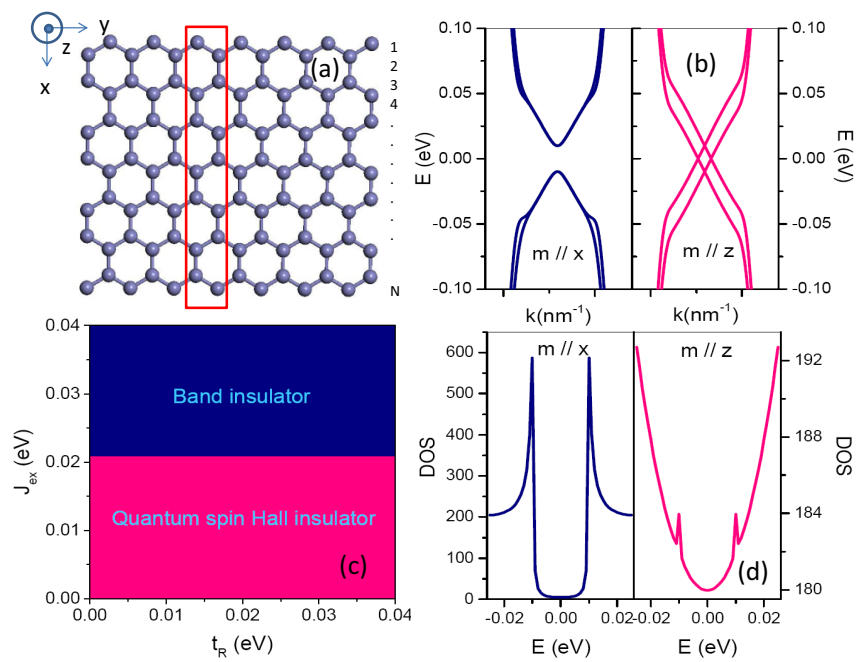

FIG. 1. (a) Top view of zigzag silicene-like nanoribbons switched by a nonmagnetic topological insulator and a magnetic topological insulator. The super unit cell is indicated by the red rectangle. (b) Band structure for different magnetization directions $\mathbf{m}$. (c) Phase diagram as a function of Rashba spin-orbit coupling and magnetic exchange. (d) Density of states for different magnetization directions. The current is directed along the $x$ axis. Parameters are $t_{\mathrm{so}}=36 \mathrm{meV}$ and $J_{\text {ex }}=10 \mathrm{meV}$. 
the spin torques and charge pumping, we first evaluate the nonequilibrium spin density $\delta \mathbf{S}$ using the Kubo formula [30],

$$
\begin{aligned}
\delta \mathbf{S}= & \frac{e \hbar}{2 \pi A} \operatorname{Re} \sum_{\mathbf{k}, a, b}\left\langle\psi_{\mathbf{k} b}|\hat{\mathbf{S}}| \psi_{\mathbf{k} a}\right\rangle\left\langle\psi_{\mathbf{k} a}|\mathbf{E} \cdot \hat{\mathbf{v}}| \psi_{\mathbf{k} b}\right\rangle \\
& \times\left[G_{\mathbf{k} b}^{R} G_{\mathbf{k} a}^{A}-G_{\mathbf{k} b}^{R} G_{\mathbf{k} a}^{R}\right]
\end{aligned}
$$

where $\mathbf{E}$ is the electric field, $\hat{\mathbf{v}}=\frac{1}{\hbar} \frac{\partial H}{\partial \mathbf{k}}$ is the velocity operator, and $G_{\mathbf{k} a}^{R}=\left(G_{\mathbf{k} a}^{A}\right)^{*}=1 /\left(E_{F}-E_{\mathbf{k} a}+i \Gamma\right) . \Gamma$ is the energy spectral broadening, and $A$ is the unit cell area. $E_{F}$ is the Fermi energy, and $E_{\mathbf{k} a}$ is the energy of electrons in band $a$. The eigenvector $\left|\psi_{\mathbf{k}, a}\right\rangle$ in band $a$ can be found by diagonalizing Eq. (10). Equation (11) contains both intraband $(a=b)$ and interband $(a \neq b)$ contributions to the nonequilibrium spin density (see the discussion in Ref. [46]). The former is related to impurity scattering and the latter only includes intrinsic contributions related to the Berry curvature at $\Gamma=0$. We ignore the vertex corrections, as they only result in a renormalization factor of the order of unity in two-dimensional hexagonal lattices [31].

For a nanoribbon in the absence of spin-orbit couping, the eigenvalues and eigenvectors around the Dirac point are independent of the magnetization direction. However, when intrinsic spin-orbit coupling is present, it acts as a valley-dependent antiferromagnetic effective field along the $z$ direction. In the low-energy limit, it reads $\sim \tau \lambda_{\mathrm{so}} \hat{\sigma}_{z} \otimes \hat{s}_{z}$. When the magnetization is directed along the $x$ axis, the cooperation of magnetic exchange and Rashba spin-orbit coupling can open up a band gap turning the system into a (trivial) band insulator, as shown in the left panel in Fig. 1(b) (see also Ref. [32]). The corresponding density of states, in the left panel in Fig. 1(d), displays an evident gap. In contrast, when the magnetization is directed along the $z$ axis, the system evolves towards the quantum spin Hall regime (insulating bulk and conducting spin-polarized edges) as shown in the right panel in Fig. 1(b). This is related to the fact that the magnetic field couples with the intrinsic spin-orbit coupling and leads to the redistribution of ground states [33]. Unlike the band insulator, the corresponding density of states shows a parabolic dependence on the energy as shown in the right panel in Fig. 1(d). For silicene-like materials, the exchange coupling is about $30 \mathrm{meV}$ [34]. In this parametric range, there are only two topological phases: trivial band insulator and quantum spin Hall insulator, as shown in Fig. 1(c). The others topological phases such as quantum anomalous Hall insulator stand beyond this parametric range.

Note that our model assumes an effective exchange Hamiltonian, the last term in Eq. (10), and disregards the actual impact of Coulomb interactions on the band structures. This assumption is expected to have a limited impact on the spincharge conversion efficiency in the metallic regime where bulk electrons dominate the transport properties of the nanoribbon. Nonetheless, Coulomb interactions are known to massively affect the topological phase transition in honeycomb systems [35]. Indeed, in graphene-like nanoribbons such interactions induce edge magnetism [36-38] and strong band structure distortion [39-41]. Most importantly for the present study, the interplay between Coulomb interaction and spin-orbit coupling dramatically alters the nature of edge states and, thereby, the transport properties at the topological phase transition (see, e.g., Refs. [42,43]). A thorough examination of the impact of strong Coulomb interaction on the spin-charge conversion efficiency in graphene-like nanoribbons would be highly valuable but remains beyond the scope of the present study.

\section{RESULTS}

\section{A. Nonequilibrium spin density and torques}

In order to understand the influence of the topological phase transition on the spin-orbit torque, we first investigate the influence of intrinsic spin-orbit coupling on the nonequilibrium spin density in a nonmagnetic nanoribbon. In this system, Rashba spin-orbit coupling enables the electrical generation of a nonequilibrium spin density, $\delta S_{y}$, an effect known as the inverse spin galvanic effect [44] and studied in detail in bulk two-dimensional hexagonal crystals [31,47]. In Fig. 2 we present the intraband [Figs. 2(a) and 2(c)] and interband [Figs. 2(b) and 2(d)] contributions to the nonequilibrium spin density in a nanoribbon as a function of the Fermi energy without [Figs. 2(a) and 2(b)] and with [Figs. 2(c) and 2(d)] intrinsic spin-orbit coupling. When the intrinsic spin-orbit coupling is absent [Figs. 2(a) and 2(b)], the system is metallic and the intraband component dominates the spin density, indicating that carriers at the Fermi surface dominate the transport. The intraband component [Fig. 2(a)] is one order of magnitude larger than the interband component [Fig. 2(b)], in agreement with the results obtained for two-dimensional graphene-like materials or two-dimensional electron gases [45-47]. When the intrinsic spin-orbit coupling is turned on [Figs. 2(c) and 2(d)], it opens up a bulk band gap and induces

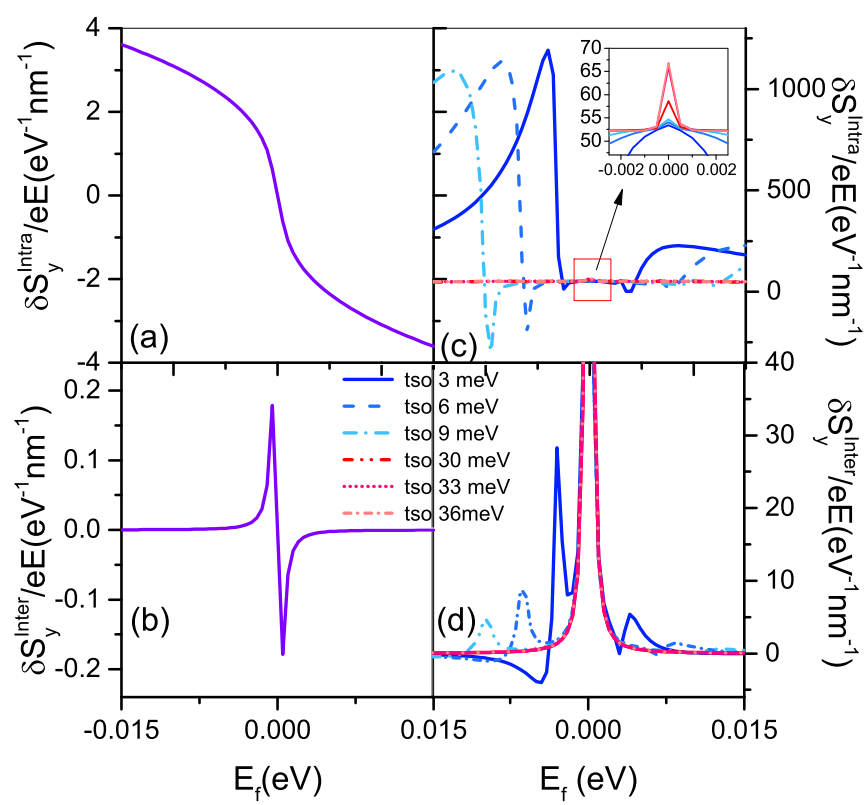

FIG. 2. Intraband and interband components of the spin density as a function of the Fermi energy in a nonmagnetic nanoribbon without $(a, b)$ and with $(c, d)$ intrinsic spin-orbit coupling. The electric field is directed along the $x$ axis. Parameters are $t_{\mathrm{so}}=0, J_{\mathrm{ex}}=0, t_{\mathrm{R}}=$ $20 \mathrm{meV}$, and $\Gamma=0.3 \mathrm{meV}$. 


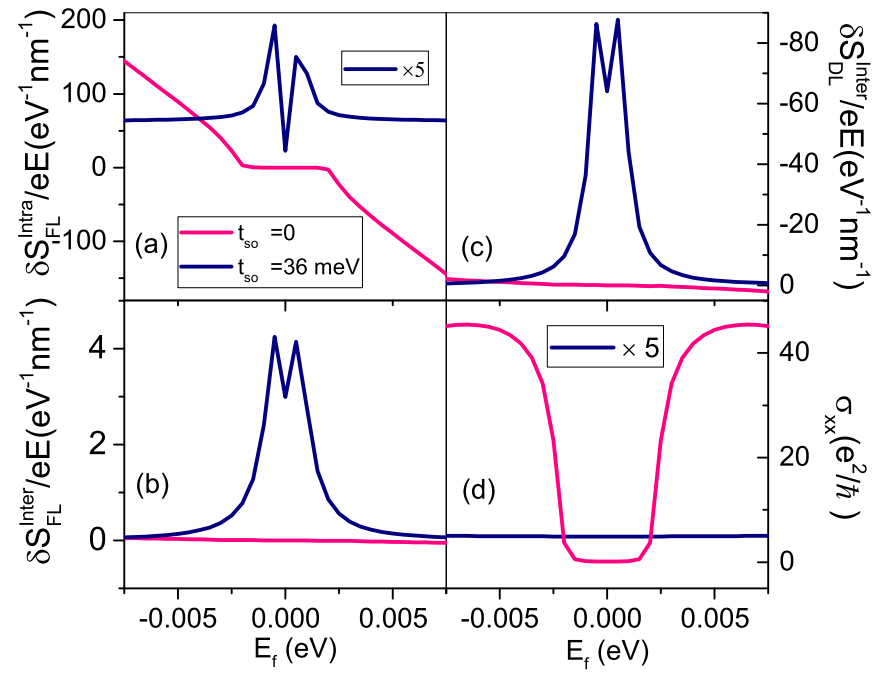

FIG. 3. (a) Intraband spin density, (b, c) interband spin density, and (d) conductance as a function of the Fermi energy without and with intrinsic spin-orbit coupling. The magnetization is directed along the $z$ axis. The exchange coupling is fixed at $J_{\mathrm{ex}}=10 \mathrm{meV}$ and other parameters are the same as in Fig. 2.

spin-polarized edge states. In the quantum spin Hall regime (low Fermi energy, no bulk transport), the intraband and interband contributions are of the same order of magnitude, while beyond the quantum spin Hall regime (high Fermi energy, edge and bulk transport coexist), the intraband contribution dominates the spin density.

Let us now turn our attention to the case of a magnetic nanoribbon. In our configuration, $\mathbf{E}=E \mathbf{x}$, and the nonequilibrium spin density can be parsed into two components,

$$
\delta \mathbf{S}=\delta S_{\mathrm{DL}} \mathbf{y} \times \mathbf{m}+\delta S_{\mathrm{FL}} \mathbf{y},
$$

referred to as the damping-like $\left(\delta S_{\mathrm{DL}}\right)$ and field-like $\left(\delta S_{\mathrm{FL}}\right)$ spin densities. We plot the field-like and the damping-like spin densities with and without intrinsic spin-orbit coupling in Fig. 3. When the intrinsic spin-orbit coupling is absent and the exchange interaction is present, the intraband component dominates the field-like spin density in Figs. 3(a) and 3(b), similarly to the case without exchange interaction displayed in Figs. 2(a) and 2(b). Moreover, the damping-like spin density [Fig. 3(c)] is lower than the field-like spin density [Fig. 3(a)] because the former is a correction arising from the precession of nonequilibrium spin density around the magnetization caused by the acceleration of carriers in the electric field $[30,46,48]$. When the intrinsic spin-orbit coupling is turned on, the nanoribbon enters the quantum spin Hall regime: transport occurs only through spin-polarized edge states, resulting in quantized conductance [Fig. 3(d)]. The intraband and interband field-like spin densities [Figs. 3(a) and 3(b)] become of comparable magnitude, while the damping-like spin density is significantly enhanced [Fig. 3(b)]. As a result, the damping-like spin density dominates over the field-like spin density. Furthermore, since the conductance is due only to edge states, the overall electrical efficiency of the torque (=torque magnitude/conductance) is dramatically enhanced in the quantum spin Hall regime.

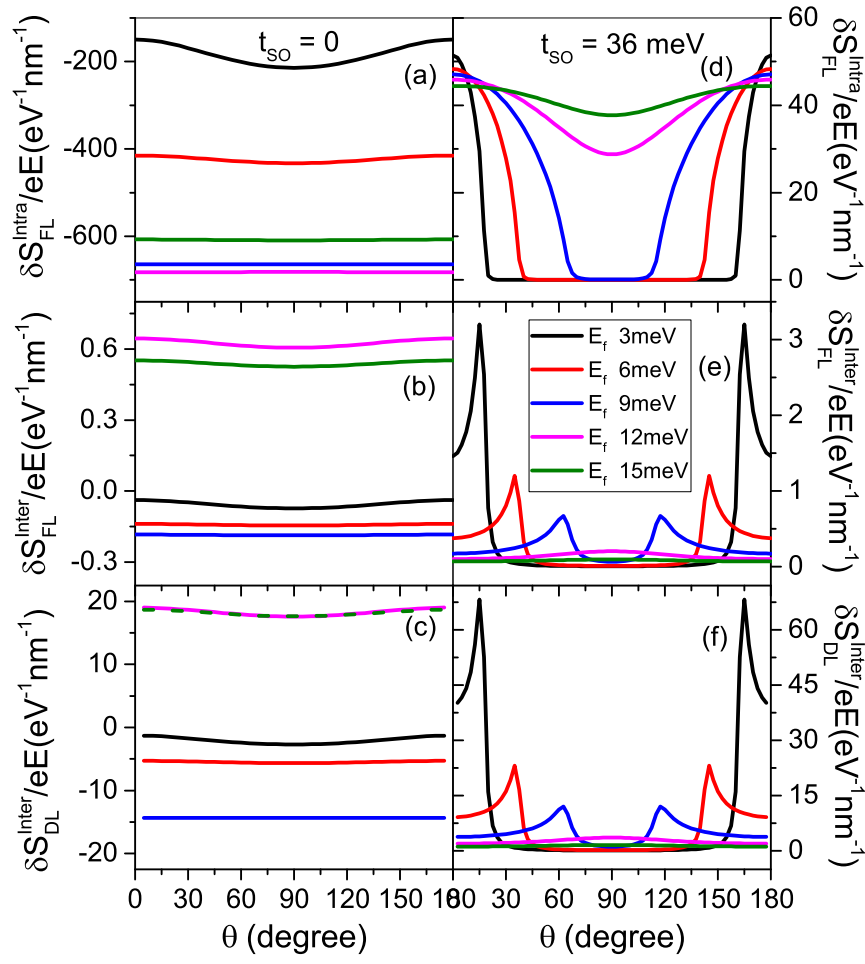

FIG. 4. Intraband and interband spin density as a function of the magnetization angle for different Fermi energies: $(a-c)$ without intrinsic spin-orbit coupling and (d-f) with intrinsic spin-orbit coupling.

The topological phase transition can be induced not only by tuning the intrinsic spin-orbit coupling but also by rotating the magnetization as shown in Figs. 1(b) and 1(c). In Fig. 4, we plot the intraband and interband contributions to the spin density as a function of the magnetization angle for different Fermi energies in the absence [Figs. 4(a)-4(c)] or presence [Figs. 4(d)-4(f)] of intrinsic spin-orbit coupling. Dramatic features can be observed depending on whether or not the nanoribbon experiences a phase transition.

When intrinsic spin-orbit coupling is absent [Figs. 4(a)4(c)] or when intrinsic spin-orbit coupling is present and the Fermi energy is high enough [ $>10 \mathrm{meV}$ in Figs. 4(d)-4(f)], the nanoribbon remains metallic independently of the magnetization direction. The spin density adopts the form given in Eq. (12) and commonly observed in two-dimensional Rashba gases [49]. Minor angular dependence is observable due to the small distortion of the Fermi surface (see also Ref. [50]). In contrast, when intrinsic spin-orbit coupling is turned on and the Fermi energy is low enough [ $<10 \mathrm{meV}$ in Figs. 4(d)-4(f)], the nanoribbon experiences a topological phase transition from the metallic $(\theta \approx 0, \pi)$ to the quantum spin Hall $(\theta \approx \pi / 2)$ regime. This transition is clearly shown in Fig. 4(d), where the intraband field-like spin density decreases dramatically (but does not vanish) upon setting the magnetization away from $\theta \approx 0, \pi$. Correspondingly, the interband damping-like and field-like contributions display an abrupt and dramatic enhancement when the magnetization angle is varied through the topological phase transition. 


\section{B. Charge pumping}

By virtue of the Onsager reciprocity, the results obtained above for the current-driven spin densities apply straightforwardly to the charge pumping through Eq. (9). From the definition of the torque, $\tau_{\mathrm{DL}}=2 J_{\mathrm{ex}} \delta S_{\mathrm{DL}} / E$ and $\tau_{\mathrm{FL}}=$ $2 J_{\mathrm{ex}} \delta S_{\mathrm{FL}} / E$, and henceforth the charge current pumped by a precessing magnetization reads

$$
\begin{aligned}
\mathbf{J}_{c}= & -\frac{2 J_{\mathrm{ex}} \mu_{B}}{d \gamma} \frac{\delta S_{\mathrm{DL}}}{E} \mathbf{z} \times\left(\mathbf{m} \times \partial_{t} \mathbf{m}\right) \\
& +\frac{2 J_{\mathrm{ex}} \mu_{B}}{d \gamma} \frac{\delta S_{\mathrm{FL}}}{E} \mathbf{z} \times \partial_{t} \mathbf{m}
\end{aligned}
$$

The first component gives both ac and dc signals [51], while the second term is purely ac. The study of nonequilibrium spin density reported above indicates that the second component, $\sim \mathbf{Z} \times \partial_{t} \mathbf{m}$, dominates in the metallic regime (since $\delta S_{\mathrm{FL}}>$ $\left.\delta S_{\mathrm{DL}}\right)$, while the first component, $\sim \mathbf{Z} \times\left(\mathbf{m} \times \partial_{t} \mathbf{m}\right)$, can be dramatically enhanced in the quantum spin Hall regime $\left(\delta S_{\mathrm{DL}}>\delta S_{\mathrm{FL}}\right)$. Furthermore, because changing the magnetization direction can induce topological phase transitions, one expects that charge pumping with the magnetization lying out of the plane of the two-dimensional nanoribbon is much more efficient than when the magnetization lies in the plane. A high charge-pumping efficiency is expected at the topological phase transition. Note, however, that the dc charge pumping vanishes when the magnetization precesses around the normal to the plane as $\left\langle\mathbf{m} \times \partial_{t} \mathbf{m}\right\rangle \equiv \mathbf{z}$.

\section{DISCUSSION AND CONCLUSION}

In summary, we have investigated the impact of the topological phase transition on the nature of the spin-orbit torque and charge pumping in quasi-one-dimensional hexagonal nanoribbons. Tuning the magnetization angle or the intrinsic spin-orbit coupling can change the system from a band insulator to a quantum spin Hall insulator. We find that the spin-charge conversion efficiencies (i.e., damping torque and charge pumping) are significantly enhanced in the quantum spin Hall regime.

Recently, a gigantic damping torque has been reported at the surface of topological insulators, with electrical efficiencies about two orders of magnitude higher than in transition metal bilayers [14]. To the best of our knowledge, no theory is currently able to explain this observation (see discussion in Ref. [12]). Although the present model does not precisely apply to the experimental case, it emphasizes that close to or in the quantum spin Hall regime, (i) the electrical efficiency of the spin-orbit torque is dramatically enhanced due to the reduction of the conductance, and, most remarkably, (ii) the damping-like torque dominates over the field-like torque. This effect, properly adapted to the case of topological insulators, could open interesting perspectives for the smart design of efficient spin-orbit interfaces through manipulation of the topological phase transition.

\section{ACKNOWLEDGMENT}

The research reported in this publication was supported by King Abdullah University of Science and Technology (KAUST).
[1] F. Ortmann, S. Roche, and S. Valenzuela, Topological Insulators: Fundamentals and Perspectives (Wiley-VCH Verlag, Weinheim, Germany, 2015).

[2] Y. L. Chen et al., Science 325, 178 (2009).

[3] S. Y. Xu et al., Science 332, 560 (2011).

[4] T. Hirahara, N. Fukui, T. Shirasawa, M. Yamada, M. Aitani, H. Miyazaki, M. Matsunami, S. Kimura, T. Takahashi, S. Hasegawa, and K. Kobayashi, Phys. Rev. Lett. 109, 227401 (2012).

[5] X. Xi, X.-G. He, F. Guan, Z. Liu, R. Zhong, J. Schneeloch, T. Liu, G. Gu, D. Xu, Z. Chen et al., Phys. Rev. Lett. 113, 096401 (2014).

[6] J. Kim, J. Kim, K.-S. Kim, and S.-H. Jhi, Phys. Rev. Lett. 109, 146601 (2012).

[7] M. Ezawa, Phys. Rev. Lett. 109, 055502 (2012).

[8] H. Pan, Z. Li, C. C. Liu, G. Zhu, Z. Qiao, and Y. Yao, Phys. Rev. Lett. 112, 106802 (2014).

[9] A. Bernevig, T. Hughes, and S. C. Zhang, Science 314, 1757 (2006).

[10] X. L. Qi, T. L. Hughes, and S. C. Zhang, Phys. Rev. B 78, 195424 (2008); I. Garate and M. Franz, Phys. Rev. Lett. 104, 146802 (2010); A. A. Burkov and D. G. Hawthorn, ibid. 105, 066802 (2010); P. Schwab, R. Raimondi, and C. Gorini, Europhys. Lett. 93, 67004 (2011).
[11] T. Yokoyama and Y. Tserkovnyak, Phys. Rev. B 89, 035408 (2014); T. Yokoyama, J. Zang, and N. Nagaosa, ibid. 81, 241410 (2010); Y. Tserkovnyak, D. A. Pesin, and D. Loss, ibid. 91, 041121 (2015).

[12] M. H. Fischer, A. Vaezi, A. Manchon, and E.-A. Kim, Phys. Rev. B 93, 125303 (2016); P. Birame Ndiaye, C. A. Akosa, M. H. Fischer, A. Vaezi, E. Kim, and A. Manchon, arXiv:1509.06929.

[13] A. R. Mellnik, J. S. Lee, A. Richardella, J. L. Grab, P. J. Mintun, M. H. Fischer, A. Vaezi, A. Manchon, E. A. Kim, N. Samarth, and D. C. Ralph, Nature 511, 449 (2014).

[14] Y. Fan, P. Upadhyaya, X. Kou, M. Lang, S. Takei, Z. Wang, J. Tang, L. He, L. T. Chang, M. Montazeri, G. Yu, W. Jiang, T. Nie, R. N. Schwartz, Y. Tserkovnyak, and K. L. Wang, Nat. Mater. 13, 699 (2014); Nat. Nanotechnol. 11, 352 (2016).

[15] Y. Wang, P. Deorani, K. Banerjee, N. Koirala, M. Brahlek, S. Oh, and H. Yang, Phys. Rev. Lett. 114, 257202 (2015).

[16] I. M. Miron et al., Nat. Mater. 9, 230 (2010); U. H. Pi et al., Appl. Phys. Lett. 97, 162507 (2010); T. Suzuki, S. Fukami, N. Ishiwata, M. Yamanouchi, S. Ikeda, N. Kasai, and H. Ohno, ibid. 98, 142505 (2011); I. M. Miron et al., Nature 476, 189 (2011).

[17] L. Liu, O. J. Lee, T. J. Gudmundsen, D. C. Ralph, and R. A. Buhrman, Phys. Rev. Lett. 109, 096602 (2012); L. Liu et al., Science 336, 555 (2012). 
[18] E. Saitoh, M. Ueda, H. Miyajima, and G. Tatara, Appl. Phys. Lett. 88, 182509 (2006); K. Ando, Y. Kajiwara, K. Sasage, K. Uchida, and E. Saitoh, IEEE Trans. Magn. 46, 1331 (2010).

[19] Z. Tang, E. Shikoh, H. Ago, K. Kawahara, Y. Ando, T. Shinjo, and M. Shiraishi, Phys. Rev. B 87, 140401(R) (2013); J. B. S. Mendes, O. Alves Santos, L. M. Meireles, R. G. Lacerda, L. H. Vilela-Leão, F. L. A. Machado, R. L. Rodríguez-Suárez, A. Azevedo, and S. M. Rezende, Phys. Rev. Lett. 115, 226601 (2015); C. Cheng et al., arXiv:1510.03451.

[20] J. C. Rojas Sanchez, L. Vila, G. Desfonds, S. Gambarelli, J. P. Attane, J. M. De Teresa, C. Magen, and A. Fert, Nat. Commun. 4, 2944 (2013).

[21] Y. Shiomi, K. Nomura, Y. Kajiwara, K. Eto, M. Novak, K. Segawa, Y. Ando, and E. Saitoh, Phys. Rev. Lett. 113, 196601 (2014); see also, C. H. Li, O. M. J. van't Erve, J. T. Robinson, Y. Liu, L. Li, and B. T. Jonker, Nat. Nanotechnol. 9, 218 (2014).

[22] M. Jamali, J. S. Lee, J. S. Jeong, F. Mahfouzi, Y. Lv, Z. Zhao, B. K. Nikolić, K. A. Mkhoyan, N. Samarth, and J.-P. Wang, Nano Lett. 15, 7126 (2015).

[23] J.-C. Rojas-Sanchez, S. Oyarzun, Y. Fu, A. Marty, C. Vergnaud, S. Gambarelli, L. Vila, M. Jamet, Y. Ohtsubo, A. Taleb-Ibrahimi, P. Le Fevre, F. Bertran, N. Reyren, J.-M. George, and A. Fert, Phys. Rev. Lett. 116, 096602 (2016).

[24] A. Brataas, Y. Tserkovnyak, G. E. W. Bauer, and P. J. Kelly, in Spin Current, edited by S. Maekawa, S. Valenzuela, E. Saitoh, and Y. Kimura (Oxford University Press, New York, 2012).

[25] F. Freimuth, S. Blügel, and Y. Mokrousov, Phys. Rev. B 92, 064415 (2015).

[26] L. Onsager, Phys. Rev. 37, 405 (1931).

[27] A. G. Swartz, P. M. Odenthal, Y. Hao, R. S. Ruoff, and R. K. Kawakami, ACS Nano 6, 10063 (2012).

[28] Z. Wang, C. Tang, R. Sachs, Y. Barlas, and J. Shi, Phys. Rev. Lett. 114, 016603 (2015).

[29] Z. Qiao, S. A. Yang, W. Feng, W. K. Tse, J. Ding, Y. Yao, J. Wang, and Q. Niu, Phys. Rev. B 82, 161414(R) (2010).

[30] H. Kurebayashi, J. Sinova, D. Fang, A. C. Irvine, J. Wunderlich, V. Novak, R. P. Campion, B. L. Gallagher, E. K. Vehstedt, L. P. Zârbo, K. Vyborny, A. J. Ferguson, and T. Jungwirth, Nat. Nanotechnol. 9, 211 (2014).

[31] A. Dyrdal and J. Barnas, Phys. Rev. B 92, 165404 (2015).
[32] Z. Qiao, H. Jiang, X. Li, Y. Yao, and Q. Niu, Phys. Rev. B 85, 115439 (2012).

[33] C. L. Kane and E. J. Mele, Phys. Rev. Lett. 95, 146802 (2005).

[34] H. X. Yang, A. Hallal, D. Terrade, X. Waintal, S. Roche, and M. Chshiev, Phys. Rev. Lett. 110, 046603 (2013).

[35] V. N. Kotov, B. Uchoa, V. M. Pereira, F. Guinea, and A. H. Castro Neto, Rev. Mod. Phys. 84, 1067 (2012).

[36] M. Fujita, K. Wakabayashi, K. Nakada, and K. Kusakabe, J. Phys. Soc. Jpn. 65, 1920 (1996).

[37] L. Matthes and F. Bechstedt, Phys. Rev. B 90, 165431 (2014).

[38] V. M. L. D. P. Goli, S. Prodhan, S. Mazumdar, and S. Ramasesha, arXiv:1601.02398v1.

[39] S.-L. Yu, X. C. Xie, and J.-X. Li, Phys. Rev. Lett. 107, 010401 (2011).

[40] J. L. Lado and J. Fernández-Rossier, Phys. Rev. Lett. 113, 027203 (2014).

[41] R. Triebl and M. Aichhorn, arXiv:1605.07855.

[42] M. Wierzbicki, J. Barna, and R. Swirkowicz, J. Phys.: Condens. Matter 27, 485301 (2015).

[43] D. Soriano and J. Fernández-Rossier, Phys. Rev. B 82, 161302(R) (2010).

[44] E. L. Ivchenko and G. E. Pikus, JETP Lett. 27, 604608 (1978); S. D. Ganichev, Int. J. Mod. Phys. B 22, 1 (2008); V. M. Edelstein, Solid State Commun. 73, 233 (1990).

[45] H. Li, X. Wang, F. Dogan, and A. Manchon, Appl. Phys. Lett. 102, 192411 (2013).

[46] H. Li, H. Gao, L. P. Zarbo, K. V. Vyborny, X. Wang, I. Garate, F. Dogan, A. Cejchan, J. Sinova, T. Jungwirth, and A. Manchon, Phys. Rev. B 91, 134402 (2015).

[47] H. Li, X. Wang, and A. Manchon, Phys. Rev. B 93, 035417 (2016).

[48] J. Sinova, D. Culcer, Q. Niu, N. A. Sinitsyn, T. Jungwirth, and A. H. MacDonald, Phys. Rev. Lett. 92, 126603 (2004).

[49] A. Manchon and S. Zhang, Phys. Rev. B 78, 212405 (2008); 79, 212405 (2009)

[50] K. S. Lee, D. Go, A. Manchon, P. M. Haney, M. D. Stiles, H. W. Lee, and K. J. Lee, Phys. Rev. B 91, 144401 (2015).

[51] H. J. Jiao and G. E. W. Bauer, Phys. Rev. Lett. 110, 217602 (2013); M. Weiler, J. M. Shaw, H. T. Nembach, and T. J. Silva, ibid. 113, 157204 (2014); D. Wei, M. Obstbaum, M. Ribow, C. H. Back, and G. Woltersdorf, Nat. Commun. 5, 3768 (2014). 